\title{
DESIGN OF A NON-SCANNING LIDAR FOR WIND VELOCITY AND DIRECTION MEASUREMENT
}

\author{
Bo Liu ${ }^{1 *}$, Zhangxian Peng ${ }^{1}$ \\ ${ }^{1}$ Institute of Optics and Electronic, CAS, CHINA, *Email: boliu@ioe.ac.cn
}

\begin{abstract}
A Doppler lidar system for wind velocity and direction measurement is presented. The lidar use a wide field of view (FOV) objective lens as an optical antenna for both beam transmitting and signal receiving. By four fibers coupled on different position on the focal plane, the lidar can implement wind vector measurement without any scanning movement.
\end{abstract}

\section{INTRODUCTION}

Lidars were employed for wind sensing since early 1970s [1]. Recently, following advances in fiber optic communications, laser wavelengths close to $1550 \mathrm{~nm}$ are used extensively in all-fiber coherent doppler wind lidars (CDL), and many commercial systems are available [2-4]. The fiber optic technology, used in optical communications industry, is employed for the generation, amplification, transmission, and manipulation of the laser beam in all-fiber CDLs [5]. For a doppler wind lidar, only radial velocity can be measured by one beam direction, to get the real wind vector, at least three beam directions are required. Most of wind lidars scan the laser beam to different directions to complete the vector measurement with a scanning device. In some cases, the mechanical scanning is not a good choice due to its slow movement, especially for those time resolution sensitive systems, such as airborne and spaceborne systems. In this paper, we discussed a design of non-scanning all-fiber wind lidar with a wide filed view optical antenna.

\section{System design}

The configuration of the system is shown in Fig. 1. The system consists of a transceiver, an optical antenna, and a signal processor. The transceiver consists of a laser source, optical couplers, frequency shifter, and pulsed fiber amplifier. The optical antenna, which consists of an optical circulator, a $1 \times 4$ fiber switch and a wide FOV objective lens. Balanced amplified photo detectors and $\mathrm{A} / \mathrm{D}$ convertors are used for signal receiving. The outgoing laser pulse can be switched to one of four fibers which are coupled on four different points on the focal plane of the objective lens. The four points are on the field of $\left(0^{\circ}, 12.5^{\circ}\right),\left(0^{\circ}\right.$,$\left.12.5^{\circ}\right),\left(12.5^{\circ}, 0^{\circ}\right)$ and $\left(-12.5^{\circ}, 0^{\circ}\right)$ respectively. By this design, the lidar can measure four different radial velocities on four directions by a fiber switch within $2 \mathrm{~ms}$. (Switching speed $0.5 \mathrm{~ms}$ )

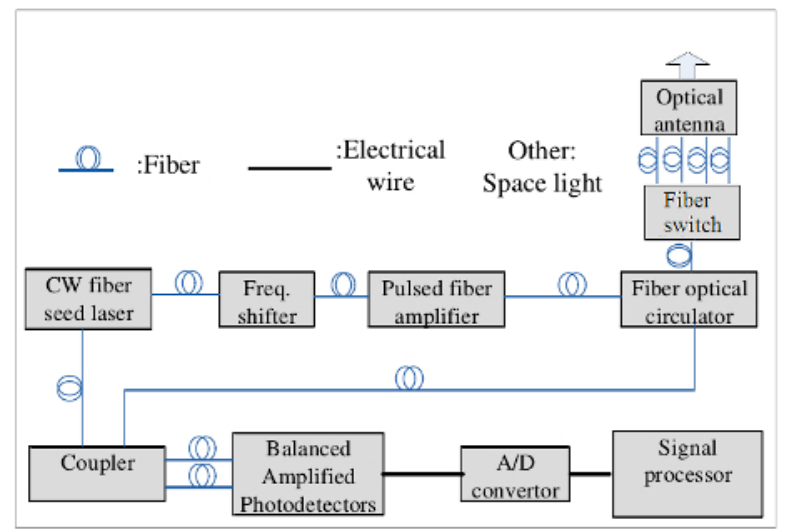

Fig.1. System configuration

Figure 2 shows the configuration of the lidar beam direction and the optics design. In a vertically setting, the four beams are pointed to the East, North, West and South at $12.5^{\circ}$ zenith angle. The objective lens consists of five pieces lens and optimized by fiber coupling efficiency. In the optical design, except for the fiber coupling efficiency, the effective receiving aperture of each point also should be taken into account. The aperture of the first lens is $76 \mathrm{~mm}$, and each beam use $91 \%$ of this aperture which means the effective receiving aperture of each point is about $69 \mathrm{~mm}$.

Figure 3 shows the system wavefront function and spot diagram of focal plane. The RMS wavefront error is 0.05 waves which is good enough for coherent detection. The RMS radius of the four points is $2.47 \mu \mathrm{m}$ and the coupling efficiency of the signal mode fiber is $53 \%$. 


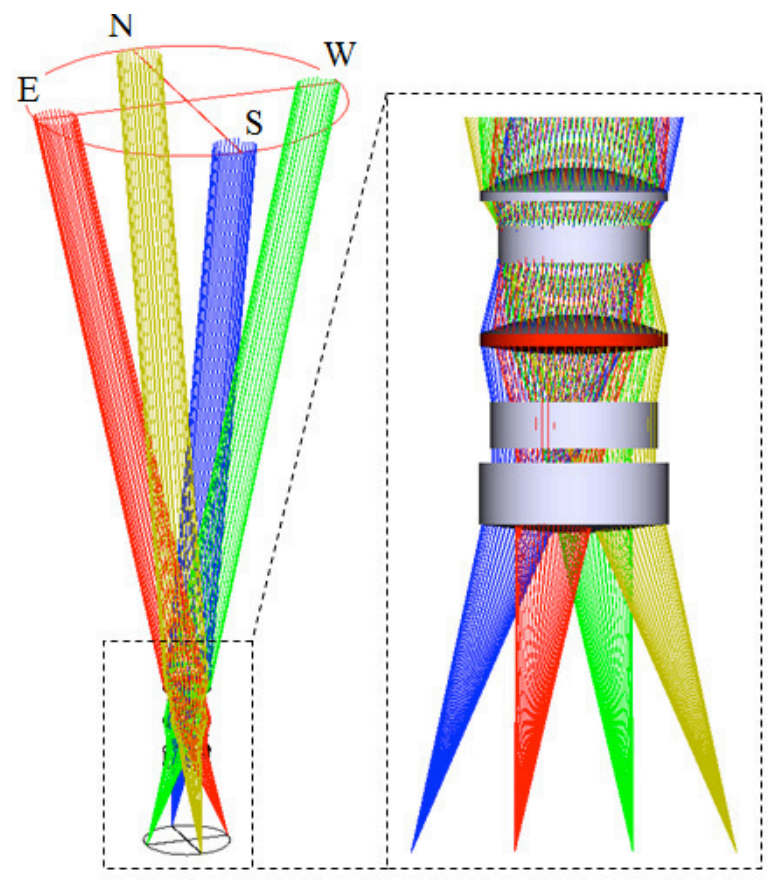

Fig.2. lidar beam direction and the optics design

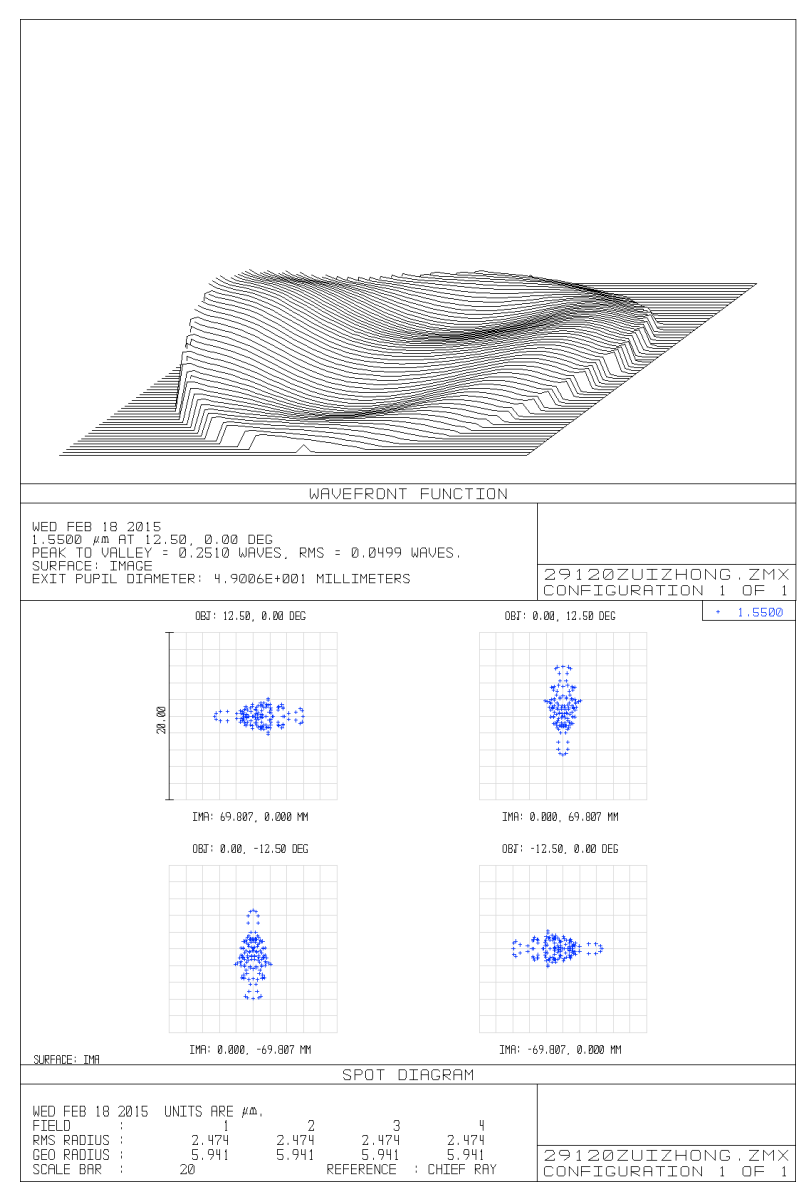

Fig.3. System wavefront function and spot diagram.

\section{Summary}

A non-scanning all-fiber pulsed wind doppler lidar was designed. The system use a wide filed view optical antenna for beam transmitting and signal receiving. Four fibers were coupled with the objective lens on four symmetrical points, and a fiber switch was used to switch the lidar beam to four directions which allow the lidar complete wind vector measurement without any scanning movement. The optics design and system configuration were presented.

\section{REFERENCES}

[1] R. M. Huffaker, A. V. Jelalian, and J. A. L. Thompson, "Laser- Doppler system for detection of aircraft trailing vortices," Proc. IEEE 58, 322326 (1970).

[2] ZephIR 300 technical specifications, (ZephIR Lidar, 2014).

[3] WINDCUBE V2, a 200m vertical wind Doppler lidar, (Leosphere, 2014).

[4] WINDAR functional specifications, (WINDAR PHOTONICS, 2014).

[5] C.F.Abari, A. T. Pedersen, and JakobMann, "An all-fiber image-reject homodyne coherent Doppler wind lidar" Opt. Express. 22(21), 25880-25894 (2014). 